\title{
Recruitment maneuvers: supine position compared with prone position in major spine surgery
}

\author{
Kieffer V., Collange O., Dieudonne M.J., Haas E., Steib A., Mertes P.M. \\ Université de Strasbourg, Dept of Anaesthesiology \& Intensive Care, \\ Strasbourg, France
}

Background and Goal of Study: Lung-protective ventilation (low tidal volumes, positive end-expiratory pressure and lung recruitment maneuvers (LRM)) has been experienced in lung (1) and abdominal major surgery (2). Thus, LRM should be use at any major surgery, especially in major spine surgery, which requiring prone position (PP). The impact of LRM performed in PP during spine surgery is currently unknown and may be different from the supine position (SP). The aim of this study was to compare hemodynamic consequences of LRM in PP to those in SP during major spine surgery.

Materials and methods: This was an observational prospective study approved by local ethics committee. Inclusion criteria were $>18$ years of age, operated of major spine surgery. Exclusion criteria were usual contraindications of LRM. LRM was defined as sustained inflation with a pressure of $30 \mathrm{cmH} 2 \mathrm{O}$ during 30 seconds. Parameters were recorded at 3 time points: after anesthesia induction in SP (T1), before incision in PP (T2) and at the end of surgery, in SP (T3) (Figure 1). Each key time was divided into: before (a), at the end of LRM (b) and 2 minutes after the LRM (c). Hemodynamics parameters (heart rate, systolic, diastolic, mean arterial pressure, pulse pressure) obtained with standard monitoring were compared with those obtained by Nexfin monitor (Edwards Lifesciences). Bayesian statistics were used to analyze the data.

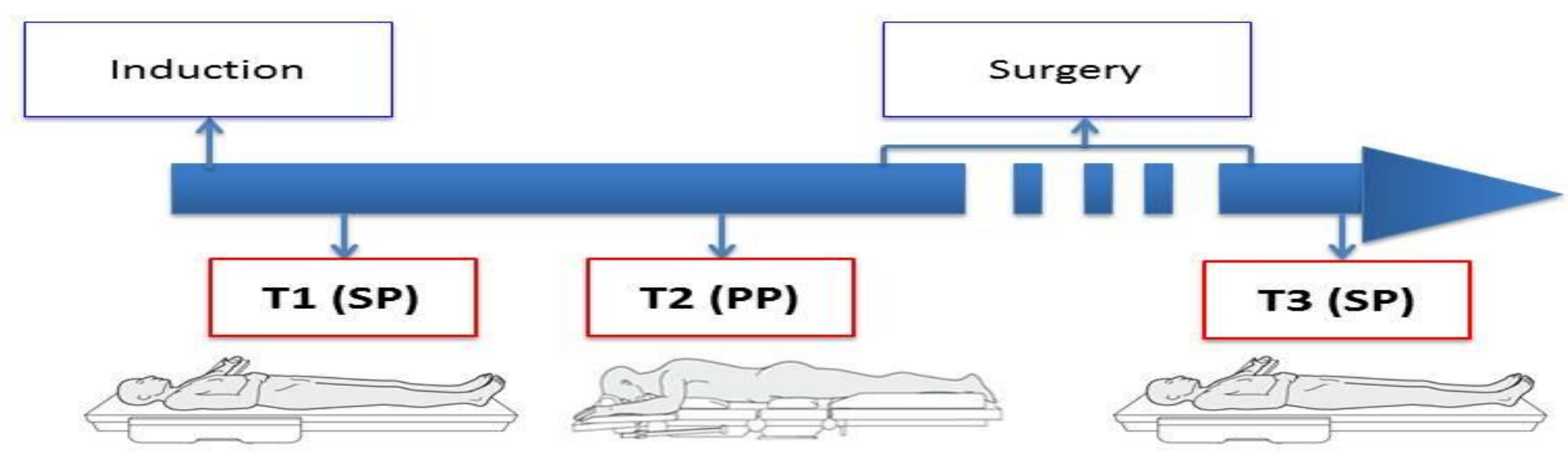

Figure 1: Description of the 3 time measurements.

Results and discussion: Fifteen patients were included from November 2014 to June 2015: 10 women (67\%), median age was 54 (19-76) years. Pulse pressure decreased during LRM (bayesian probability $(\mathrm{pr})=100 \%)$. The decrease in pulse pressure was significantly more pronounced in PP (T2: $18 \pm 2$ $\mathrm{mm} \mathrm{Hg}$ ) compared to $\mathrm{SP}$ (T1 and T3, respectively: $13 \pm 2$ $\mathrm{mmHg}$ and $13 \pm 2 \mathrm{mmHg}$; pr $\geq 93 \%$ ) (Figure 2).

We hypothesized that this could reflect a more pronounced stroke volume decrease in PP. Other hemodynamic parameters were similarly decreased in PP and SP during LRM. Correlation coefficients between standard and Nexfin monitors for systolic, diastolic and mean pressure were: 0.92 (0.87-0.98), $0.87(0.80-0.94)$ and $0.90(0.85-0.96)$.

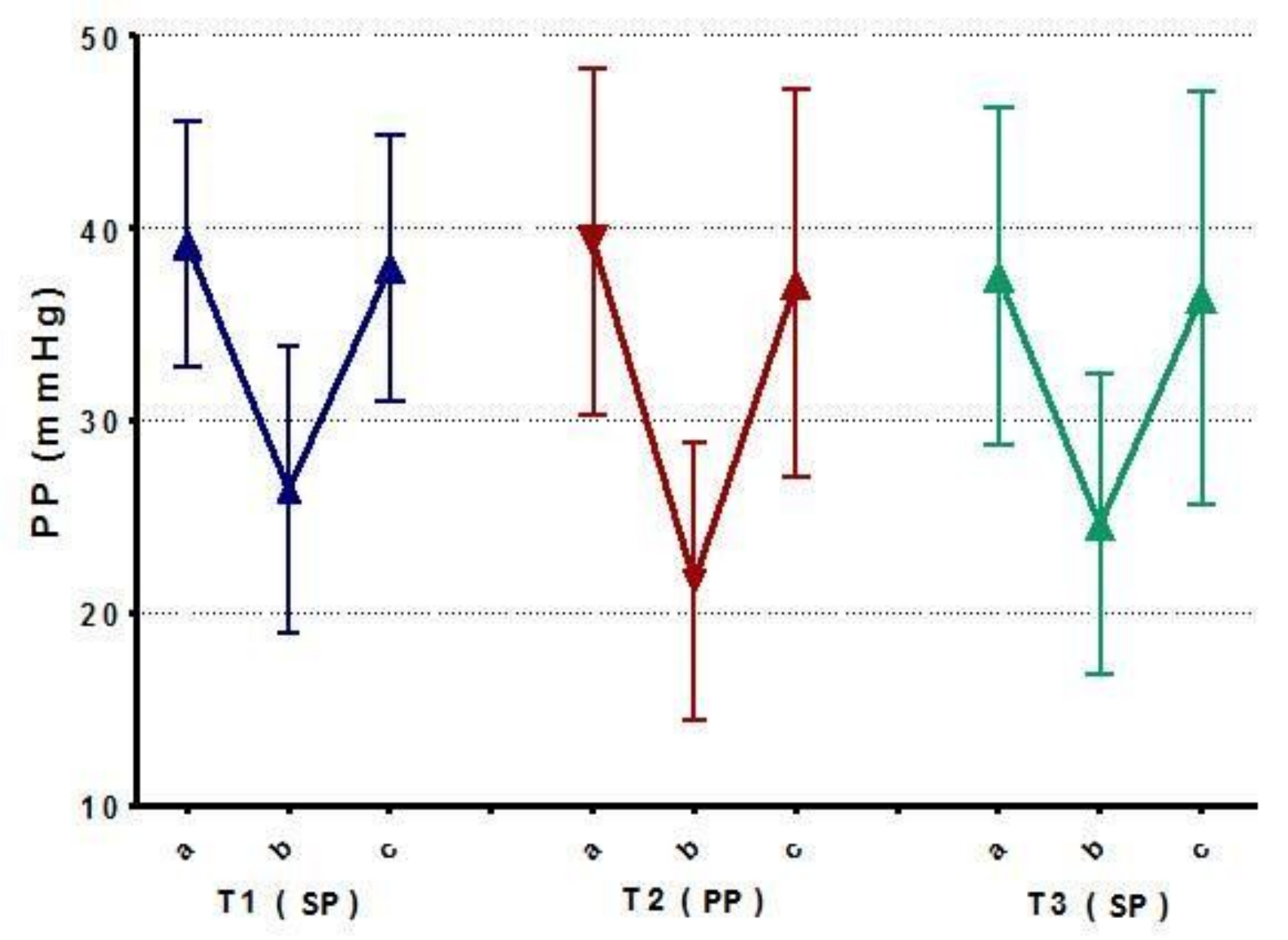

Figure 2: PP variations at the 3 time points (subdivided into: $\mathrm{a}, \mathrm{b}$ and $\mathrm{c}$ ).

Conclusion(s): During major spine surgery and compared to SP, LRM performed in PP showed a more pronounced decrease in arterial pulse pressure and probably in stroke volume. 